\title{
Stability and D-stability for Switched Positive Systems*
}

\author{
Oliver Mason, Vahid S. Bokharaie, Robert Shorten
}

\begin{abstract}
We consider a number of questions pertaining to the stability of positive switched linear systems. Recent results on common quadratic, diagonal, and copositive Lyapunov function existence are reviewed and their connection to the stability properties of switched positive linear systems is highlighted. We also generalise the concept of D-stability to positive switched linear systems and present some preliminary results on this topic.
\end{abstract}

\section{Introduction}

While the stability properties of positive linear time-invariant (LTI) systems have been thoroughly investigated and are now completely understood, the theory for nonlinear, uncertain and time-varying positive systems is considerably less welldeveloped. In fact, many natural and fundamental questions on the stability of such systems remain unanswered. It is clear that for many practical applications there is a need to extend the theory for positive LTI systems to broader and more realistic system classes incorporating nonlinearities and time-varying parameters. Another

Oliver Mason

Hamilton Institute, National University of Ireland Maynooth, Co. Kildare, Ireland e-mail: oliver.mason@nuim.ie

Vahid Bokharaie

Hamilton Institute, National University of Ireland Maynooth, Co. Kildare, Ireland e-mail: vahid.bokharaie@nuim.ie

Robert Shorten

Hamilton Institute, National University of Ireland Maynooth, Co. Kildare, Ireland e-mail: robert.shorten@nuim.ie

* Supported by the Irish Higher Education Authority (HEA) PRTLI Network Mathematics grant, by Science Foundation Ireland (SFI) grant 08/RFP/ENE1417 and by SFI PI Award 07/IN.1/1901. 
separate and interesting line of recent research has focussed on extending the stability properties of positive LTI systems to positive descriptor systems [1].

Our principal focus in the present paper is on extending the stability theory of positive LTI systems to switched positive linear systems [2]. We review recent work on the stability of these systems, highlighting the connection between various notions of stability and the existence of corresponding types of common Lyapunov function. We also consider an extension of the concept of D-stability to positive switched linear systems, present some preliminary results for this question and highlight some directions for future research.

\section{Notation and Background}

Throughout, $\mathbb{R}$ denotes the field of real numbers, $\mathbb{R}_{+}$denotes the set of non-negative real numbers, $\mathbb{R}^{n}$ stands for the vector space of all $n$-tuples of real numbers and $\mathbb{R}^{m \times n}$ is the space of $m \times n$ matrices with real entries. For $x$ in $\mathbb{R}^{n}, x_{i}$ denotes the $i^{\text {th }}$ component of $x$, and the notation $x \succ 0(x \succeq 0)$ means that $x_{i}>0\left(x_{i} \geq 0\right)$ for $1 \leq i \leq n$. The notations $x \prec 0$ and $x \preceq 0$ are defined in the obvious manner.

We write $A^{T}$ for the transpose of $A \in \mathbb{R}^{n \times n}$ and for a symmetric $P$ in $\mathbb{R}^{n \times n}$ the notation $P>0$ means that the matrix $P$ is positive definite.

Throughout the paper, in an abuse of notation, for LTI systems we shall use the term stability to denote asymptotic stability. Also, when referring to switched linear systems, stability shall be used to denote asymptotic stability under arbitrary switching [2].

For a positive LTI system

$$
\dot{x}(t)=A x(t)
$$

where $A \in \mathbb{R}^{n \times n}$ is a Metzler matrix (meaning that the off-diagonal entries of $A$ are non-negative), the equivalences we collect in the following result are well known.

Proposition 1. [3] Let $A \in \mathbb{R}^{n \times n}$ be a Metzler matrix. The following statements are equivalent:

(a)The LTI system (1) is stable;

(b)A is Hurwitz, meaning that its eigenvalues lie in the open left half plane;

(c) There exists $P>0$ such that $A^{T} P+P A<0$;

(d)There exists a diagonal matrix $D>0$ such that $A^{T} D+D A<0$;

(e) There exists a vector $v \succ 0$ in $\mathbb{R}^{n}$ with $A v \prec 0$;

(f) For any diagonal matrix $D>0$, the system $\dot{x}(t)=D A x(t)$ is stable.

While the equivalence of (a), (b) and (c) in the previous result also holds for any LTI system, properties (d), (e) and (f) are specific to positive LTI systems. With regard to point (e), as $A$ is Hurwitz and Metzler if and only if $A^{T}$ is Hurwitz, an equivalent condition for stability for positive LTI systems is the existence of $v \succ 0$ 
satisfying $A^{T} v \prec 0$. Such a $v$ can be used to define a copositive linear Lyapunov function $V(x)=v^{T} x$ for the system (1).

The property described in (f) is known as $D$-stability and establishes that stability of positive LTI systems is robust with respect to parametric uncertainties given by diagonal scaling. Later in the paper, we shall be concerned with investigating the connection between concepts similar to those in (e) and (f) for switched positive linear systems. Before this, in the following section, we shall review some recent work on the stability of switched positive linear systems.

\section{Lyapunov Functions and Stability for Switched Positive Linear Systems}

It is well known that a switched positive linear system of the form

$$
\dot{x}(t)=A(t) x(t) \quad A(t) \in\left\{A_{1}, A_{2}\right\}
$$

can be unstable for certain choices of switching sequence even when the individual system matrices $A_{1}, A_{2}$ are asymptotically stable [2]. This observation has led to great interest in the stability of such systems under arbitrary switching regimes. A key result in this connection is that stability of (2) is equivalent to the existence of a common Lyapunov function for the individual component LTI systems [2]. In the light of Proposition 1, three classes of Lyapunov function naturally suggest themselves for positive switched linear systems:

- Common Quadratic Lyapunov Functions (CQLFs): $V(x)=x^{T} P x$ where $P=$ $P^{T}>0$ and $A_{i}^{T} P+P A_{i}<0$ for $i=1,2$;

- Common Diagonal Lyapunov Functions (CDLFs): $V(x)=x^{T} D x$ where $D=$ $\operatorname{diag}\left(d_{1}, \ldots, d_{n}\right), D>0$ and $A_{i}^{T} D+D A_{i}<0$ for $i=1,2$;

- Common Linear Copositive Lyapunov Functions (CLLFs): $V(x)=v^{T} x$ where $v \succ 0$ and $A_{i}^{T} v \prec 0$ for $i=1,2$.

In the interests of brevity, we shall abuse notation slightly and say that the matrices $A_{1}, A_{2}$ have a CQLF, CDLF or CLLF rather than always referring to the associated LTI systems.

Recall the following well-known necessary condition for the stability of positive switched linear systems (in fact this is a necessary condition for stability for general switched linear systems)[2].

Lemma 1. Let $A_{1}, A_{2} \in \mathbb{R}^{n \times n}$ be Metzler and Hurwitz. Suppose that the associated switched positive linear system (2) is stable. Then for any real $\gamma \geq 0, A_{1}+\gamma A_{2}$ is Hurwitz.

Common Quadratic Lyapunov Functions (CQLFs)

In [4], the relationship between the existence of CQLFs, the stability of all matrices of the form $A_{1}+\gamma A_{2}$ with $\gamma \geq 0$, and the stability of the system (2) was considered. For 2-dimensional systems, the following result was established. 
Theorem 1. Let $A_{1}, A_{2} \in \mathbb{R}^{2 \times 2}$ be Hurwitz and Metzler. Then the following statements are equivalent:

(a) $A_{1}, A_{2}$ have a CQLF;

(b)The switched system (2) is stable;

(c) $A_{1}+\gamma A_{2}$ is Hurwitz for all real $\gamma \geq 0$.

Further, the equivalence of (b) and (c) can be extended to the case of an arbitrary finite number of positive LTI systems. Formally, it was shown in [4] that given Metzler, Hurwitz matrices $A_{1}, \ldots, A_{k}$ in $\mathbb{R}^{2 \times 2}$, the switched system $\dot{x}(t)=A(t) x(t)$, $A(t) \in\left\{A_{1}, \ldots, A_{k}\right\}$ is stable if and only if $A_{1}+\gamma_{2} A_{2}+\cdots+\gamma_{k} A_{k}$ is Hurwitz for all real $\gamma_{2} \geq 0, \ldots \gamma_{k} \geq 0$.

The equivalence of (a), (b) and (c) fails immediately for 3-dimensional systems. Moreover, the equivalence of (b) and (c) is not true for arbitrary dimensions [4]. In fact, in a very recent paper [5], a 3-dimensional example of an unstable switched system for which $A_{1}+\gamma A_{2}$ was Hurwitz for all $\gamma \geq 0$ was explicitly described. In connection with CQLF existence and the stability of positive switched linear systems, it has been shown in [6] for 2 and 3 dimensional systems that if $\operatorname{rank}\left(A_{2}-A_{1}\right)=1$, and $A_{2}, A_{1}$ are both Hurwitz, then the associated LTI systems always possess a CQLF and the switched linear system (2) is stable.

Common Diagonal Lyapunov Functions (CDLFs)

As stable positive LTI systems have diagonal Lyapunov functions, it is natural to ask under what conditions families of such systems will possess a common diagonal Lyapunov function. In the paper [7], the following result was derived for systems with irreducible system matrices (for the definition of irreducible matrices, see [8]).

Theorem 2. Let $A_{1}, A_{2} \in \mathbb{R}^{n \times n}$ be irreducible, Metzler and Hurwitz. $A_{1}$, $A_{2}$ have a $C D L F$ if and only if $A_{1}+D A_{2} D$ is Hurwitz for all diagonal matrices $D>0$.

The above result allows us to establish a connection between the existence of a CDLF and a form of robust stability for switched positive linear systems. First of all, note that for $A_{1}, A_{2}$ irreducible, Metzler and Hurwitz, Theorem 2 shows that if $A_{1}, A_{2}$ have a CDLF, then so do $D_{1} A_{1} D_{1}, D_{2} A_{2} D_{2}$ for any choice of diagonal matrices $D_{1}>0, D_{2}>0$. Hence the existence of a CDLF guarantees the stability of the positive switched linear system

$$
\dot{x}(t)=A(t) x(t) \quad A(t) \in\left\{D_{1} A_{1} D_{1}, D_{2} A_{2} D_{2}\right\}
$$

for any diagonal matrices $D_{1}>0, D_{2}>0$.

Conversely, if $A_{1}, A_{2}$ do not have a CDLF, then it follows from Theorem 2 that there is some diagonal matrix $D>0$ such that $A_{1}+D A_{2} D$ is not Hurwitz. This then immediately implies from Lemma 1 that the switched system (3) is not stable with $D_{1}=I$, and $D_{2}=D$. This discussion establishes the following result.

Proposition 2. Let $A_{1}, A_{2} \in \mathbb{R}^{n \times n}$ be irreducible, Metzler and Hurwitz. The switched system (3) is stable for any diagonal matrices $D_{1}>0, D_{2}>0$ if and only if $A_{1}, A_{2}$ have a CDLF. 


\section{Common Linear Copositive Lyapunov Functions (CLLFs)}

It is also possible to establish the stability of positive switched linear systems using copositive linear Lyapunov functions. As noted in [9], traditional Lyapunov functions may give conservative stability conditions for positive switched systems as they fail to take into account that trajectories are naturally constrained to the positive orthant. The existence of a CLLF for a pair of Metzler, Hurwitz matrices $A_{1}, A_{2}$ is equivalent to the feasibility of the linear inequalities $v \succ 0, A_{1}^{T} v \prec 0, A_{2}^{T} v \prec 0$. For the most part, we shall be concerned with the feasibility of the related system of inequalities $v \succ 0, A_{1} v \prec 0, A_{2} v \prec 0$ as this is more relevant to the extension of the concept of D-stability for switched positive linear systems that interests us. Conditions for the feasibility of this system of inequalities (for compact sets of matrices) have been given in terms of P-matrix sets in the paper [10].

It is important for what follows to make clear the distinction between the existence of a common $v \succ 0$ satisfying $A_{i}^{T} v \prec 0$ for $i=1,2$ (CLLF existence), and the existence of a common $v \succ 0$ such that $A_{i} v \prec 0$ for $i=1,2$. For switched systems (in contrast with the LTI case), these two conditions are not equivalent. This can be seen from the following simple $2 \times 2$ example.

Example 1.

$$
A_{1}=\left(\begin{array}{cc}
-1 & 2 \\
1 & -3
\end{array}\right), A_{2}=\left(\begin{array}{cc}
-6 & 6 \\
2 & -6
\end{array}\right)
$$

It can be verified algebraically that $A_{i} v \prec 0$ for $i=1,2$ where $v=\left(\begin{array}{ll}5 & 2\end{array}\right)^{T}$. However, it is easy to show that there can be no $v \succ 0$ satisfying $A_{i}^{T} v \prec 0$ for $i=1,2$.

An algebraic condition for CLLF existence was derived in [11]. In the interests of brevity, we shall not explicitly state this result here but rather state the following technical result which follows from Theorem 3.1 in that paper. This fact shall prove useful in our later discussion.

Lemma 2. Let $A_{1}, A_{2} \in \mathbb{R}^{n \times n}$ be Metzler and Hurwitz. Suppose that there is no nonzero $v \succeq 0$ in $\mathbb{R}^{n}$ with $A_{1} v \preceq 0, A_{2} \preceq 0$. Then there is some diagonal $D>0$ such that $A_{1}+D A_{2}$ is singular.

\section{Switched Positive Linear Systems and D-Stability: The 2-d Case}

In this and the following section, we shall investigate the following generalisation of the notion of D-stability to positive switched linear systems.

Definition 1. Let $A_{1}, A_{2} \in \mathbb{R}^{n \times n}$ be Metzler and Hurwitz. The associated switched positive linear system (2) is said to be $\mathrm{D}$-stable if for any diagonal matrices $D_{1}, D_{2} \in$ $\mathbb{R}^{n \times n}$ with $D_{1}>0, D_{2}>0$, the system

$$
\dot{x}(t)=A(t) x(t) \quad A(t) \in\left\{D_{1} A_{1}, D_{2} A_{2}\right\}
$$


is stable.

For positive LTI systems, Proposition 1 shows that stability and D-stability are equivalent. Our first observation, in Example 2, is to note that this equivalence is not true in the switched case. First of all, we note the following simple necessary condition for D-stability, which follows immediately from Lemma 1.

Lemma 3. Let $A_{1}, A_{2} \in \mathbb{R}^{n \times n}$ be Metzler and Hurwitz. Suppose that the associated switched positive linear system (2) is D-stable. Then for any diagonal matrix $D>0$, $A_{1}+D A_{2}$ is Hurwitz.

Example 2. Consider the Metzler, Hurwitz matrices in $\mathbb{R}^{2 \times 2}$

$$
A_{1}=\left(\begin{array}{cc}
-2 & 0 \\
1 & -4
\end{array}\right), A_{2}=\left(\begin{array}{cc}
-1 & 5 \\
0 & -1
\end{array}\right)
$$

It is straightforward to verify that $A_{1}+\gamma A_{2}$ is Hurwitz for all $\gamma \geq 0$. Hence by Theorem 1, the associated switched system is stable. On the other hand, choosing

$$
D=\left(\begin{array}{cc}
20 & 0 \\
0 & 0.5
\end{array}\right)
$$

it is easily verified that $A_{1}+D A_{2}$ is not Hurwitz. Hence by Lemma 3 the associated switched system is not D-stable.

The above example illustrates that for switched positive linear systems, the concepts of stability and D-stability are not equivalent, in contrast to the LTI system case. In the following result, we show that the necessary condition given in Lemma 3 is also sufficient for D-stability for 2-dimensional systems.

Theorem 3. Let $A_{1}, A_{2} \in \mathbb{R}^{2 \times 2}$ be Metzler and Hurwitz. The positive switched linear system (2) is D-stable if and only if $A_{1}+D A_{2}$ is Hurwitz for all diagonal matrices $D>0$.

Proof: Lemma 3 has already established the necessity of this condition. For sufficiency let $D_{1}>0, D_{2}>0$ be diagonal matrices and let $\gamma \geq 0$ be any non-negative real number. By hypothesis, $A_{1}+\gamma D_{1}^{-1} D_{2} A_{2}$ is Hurwitz for $\gamma>0$ and it is trivially true for $\gamma=0$. However, this matrix is also Metzler and hence by point (f) of Proposition 1, $D_{1} A_{1}+\gamma D_{2} A_{2}=D_{1}\left(A_{1}+\gamma D_{1}^{-1} D_{2} A_{2}\right)$ is also Hurwitz. It now follows immediately from Theorem 1 that the switched system (4) associated with $D_{1} A_{1}, D_{2} A_{2}$ is stable. As this is true for any diagonal $D_{1}>0, D_{2}>0$, the system (2) is D-stable.

The next result establishes a connection between the existence of a common solution to the inequalities $v \succ 0, A_{i} v \prec 0$ for $i=1,2$ and D-stability for (2).

Corollary 1. Let $A_{1}, A_{2} \in \mathbb{R}^{2 \times 2}$ be Metzler and Hurwitz. Then:

(i) If there is some $v \succ 0$ with $A_{1} v \prec 0, A_{2} v \prec 0$ then the system (2) is D-stable;

(ii)If (2) is D-stable then there exists some non-zero $v \succeq 0$ with $A_{1} v \preceq 0, A_{2} v \preceq 0$. 
Proof: (i) Suppose there is some $v \succ 0$ with $A_{i} v \prec 0$ for $i=1,2$. Then for any diagonal $D>0, D A_{2} v \prec 0$ and $\left(A_{1}+D A_{2}\right) v \prec 0$. Moreover, $A_{1}+D A_{2}$ is Metzler. Hence, from point (e) of Proposition 1, it follows that $A_{1}+D A_{2}$ is Hurwitz. Theorem 3 now implies that the switched system (2) is D-stable.

(ii) If (2) is D-stable, then Theorem 3 implies that $A_{1}+D A_{2}$ is Hurwitz for all diagonal $D>0$. It now follows from Lemma 2 that there must exist some non-zero $v \succeq 0$ with $A_{1} v \preceq 0, A_{2} v \preceq 0$.

Note that the sufficient condition for D-stability presented in point (i) of Corollary 1 is not necessary as demonstrated by the following example.

Example 3. Consider the Metzler, Hurwitz matrices $A_{1}, A_{2}$ given by:

$$
A_{1}=\left(\begin{array}{cc}
-2 & 1 \\
2 & -2
\end{array}\right), A_{2}=\left(\begin{array}{cc}
-3 & 1 \\
2 & -1
\end{array}\right)
$$

Using Theorem 4.1 of [11] it is straightforward to show that there is no vector $v \succ 0$ with $A_{1} v \prec 0, A_{2} v \prec 0$. On the other hand, it can be verified algebraically that for any diagonal $D>0, A_{1}+D A_{2}$ is Hurwitz and hence the switched system (2) is D-stable by Theorem 3 .

\section{D-Stability in Higher Dimensions}

In this section, we present a result extending Corollary 1 to higher dimensional positive switched linear systems. While the following result is stated for switched systems with two constituent systems, the argument can easily be amended to derive a corresponding result for an arbitrary number of constituent systems.

Theorem 4. Let $A_{1}, A_{2} \in \mathbb{R}^{n \times n}$ be Metzler and Hurwitz. Then:

(i) If there is some $v \succ 0$ with $A_{1} v \prec 0, A_{2} v \prec 0$ then the system (2) is D-stable; (ii)If (2) is D-stable then there exists some non-zero $v \succeq 0$ with $A_{1} v \preceq 0, A_{2} v \preceq 0$.

Proof:

(i)

The first step in proving (i) is to show that the existence of such a $v$ is sufficient for the stability of the switched system (2). With this in mind, suppose that there exists some $v \succ 0$ satisfying $A_{1} v \prec 0, A_{2} v \prec 0$, and let a (piecewise-constant) switching signal $\sigma: \mathbb{R}_{+} \rightarrow\{1,2\}$ be given such that $A(t)=A_{\sigma(t)}$ for all $t \geq 0$. Furthermore, let $0=t_{0}, t_{1}, t_{2}, \ldots, t_{k}, \ldots$, be the switching times or points of discontinuity of $\sigma$. As is standard for switching systems [2], we assume that there is some non-vanishing dwell-time $\tau>0$ such that $t_{k+1}-t_{k} \geq \tau$ for all $k \geq 0$. Let $x^{\sigma}\left(., x_{0}\right)$ denote the unique, piecewise $C^{1}$ solution of (2) corresponding to the initial condition $x_{0}$ and the switching signal $\sigma$. Also, for $i=1,2$, let $x^{(i)}\left(., x_{0}\right)$ denote the unique solution of the stable positive LTI system $\dot{x}=A_{i} x$ corresponding to the initial state $x_{0}$.

Note the following readily verifiable facts concerning the solutions of the positive LTI systems with system matrices $A_{1}, A_{2}$. 
(a)For $i=1,2$, if $x_{0} \succeq 0, x_{1} \succeq 0$ satisfy $x_{0} \preceq x_{1}$, then $x^{(i)}\left(t, x_{0}\right) \preceq x^{(i)}\left(t, x_{1}\right)$ for all $t \geq 0$. This simply records the well-known fact that positive LTI systems are monotone;

(b)For $i=1,2$, as $\frac{d}{d t} x^{(i)}(0, v)=A_{i} v \prec 0$, it follows that there is some $\delta>0$ such that $x^{(i)}(t, v) \prec v$ for $0 \leq t \leq \delta$.

Combining (a) and (b) we see immediately that for $0 \leq t \leq \delta$, and $i=1,2$,

$$
x^{(i)}(t+\delta, v)=x^{(i)}\left(t, x^{(i)}(\delta, v)\right) \preceq x^{(i)}(t, v) \preceq v .
$$

Simply iterating this process, it is easy to see that for $i=1,2, x^{(i)}(t, v) \preceq v$ for all $t \geq 0$.

Now consider the solution $x^{\sigma}(., v)$ of (2) corresponding to the initial condition $v$ and the switching signal $\sigma$. The argument in the previous paragraph guarantees that for $0 \leq t \leq t_{1}, x^{\sigma}(t, v) \preceq v$ (as the dynamics in this interval are given by one of the constituent positive LTI systems). But in the second interval $\left[t_{1}, t_{2}\right)$, the system dynamics are again given by a positive LTI system with $x^{\sigma}\left(t_{1}, v\right) \preceq v$ as initial condition. Hence from the previous argument combined with point (a) above, we can conclude that for $t_{1} \leq t \leq t_{2}, x^{\sigma}(t, v) \preceq v$. Continuing in this way, we can easily see that for all $t \geq 0$, we have $x^{\sigma}(t, v) \preceq v$. As the switching signal $\sigma$ was arbitrary, we can conclude that $x^{\sigma}(t, v) \preceq v$ holds for all switching signals.

It is now straightforward to show that the solutions of (2) are uniformly bounded. In fact, for any $x_{0} \succeq 0$ such that $\left\|x_{0}\right\|_{\infty} \leq K_{1}$, if $v_{\text {min }}=\min \left\{v_{1}, \ldots, v_{n}\right\}$, then $x_{0} \preceq$ $\left(K_{1} / v_{\text {min }}\right) v$. It now follows that for all $t \geq 0, x^{\sigma}\left(t, x_{0}\right) \preceq\left(K_{1} / v_{\text {min }}\right) v$ and hence that $\left\|x^{\sigma}\left(t, x_{0}\right)\right\|_{\infty} \leq K_{1}\left(v_{\max } / v_{\min }\right)$ for all $t \geq 0$ where $v_{\max }=\max \left\{v_{1}, \ldots, v_{n}\right\}$.

Now if there is some $v \succ 0$ with $A_{i} v \prec 0$ for $i=1,2$, then $\left(A_{i}+\varepsilon I\right) v \prec 0$ for sufficiently small positive $\varepsilon>0$. Therefore, the trajectories of the switched system corresponding to $A_{1}+\varepsilon I, A_{2}+\varepsilon I$ are also uniformly bounded for small enough positive $\varepsilon>0$. This immediately implies that the original system (2) is globally asymptotically stable.

To complete the proof of (i), note that for any positive definite diagonal matrices $D_{1}, D_{2}$, the matrices $D_{1} A_{1}$ and $D_{2} A_{2}$ are Metzler and Hurwitz. Moreover, if $A_{i} v \prec$ 0 for $i=1,2$, then $D_{i} A_{i} v \prec 0$ for $i=1,2$. The above argument now immediately implies that the system (4) is stable and hence the original system (2) is D-stable as claimed.

The result given by (ii) follows immediately from Lemma 3 and Lemma 2.

Note that the result given by (i) provides a condition for stability of (2) that is distinct although related to the condition given by CLLF existence.

\section{Concluding remarks}

In this paper, we have discussed a number of problems relating to the stability properties of switched positive linear systems. In particular, we have reviewed recent work on common quadratic, copositive and diagonal Lyapunov functions for these 
systems and on the relationship between the existence of such functions and various notions of stability for switched positive systems. We have also discussed the notion of D-stability for positive switched systems and presented separate necessary and sufficient conditions for D-stability for $n$-dimensional systems. More detailed and complete results have also been given for 2-dimensional systems.

A number of interesting directions for future research emerge from the work described here. For instance, it would be interesting to investigate the possibility of Theorem 3 extending to dimensions higher than 2, even for some restricted system class. Also, the question of whether stability and D-stability are equivalent for any subclass of positive switched linear systems arises naturally. It is straightforward to show that this is true for upper (or lower) triangular positive systems, for example, but are there any more interesting such classes?

\section{Acknowledgements}

The authors are very grateful to the organisers of the session on Stability and Control of Positive Systems for their kind invitation to contribute to the session.

\section{References}

1. Virnik, E. Stability Analysis of Positive Descriptor Systems. Linear Algebra and its Applications, 429:2640-2659, 2008.

2. Shorten R., Wirth F., Mason O., Wulff K., King C. Stability Theory for Switched and Hybrid Systems SIAM Review, 49(4):545-592, 2007.

3. Farina L., Rinaldi S. (2000) Positive Linear Systems. Theory and Applications. Pure and Applied mathematics. John Wiley \& Sons, Inc., New York

4. Gurvits L., Shorten R., Mason O. On the Stability of Switched Positive Linear Systems. IEEE Transactions on Automatic Control, 52(6):1099-1103, 2007.

5. Fainshil L., Margaliot M., Chigansky P. Positive Switched Linear Systems are not Uniformly Stable, even for $\mathrm{n}=3$ IEEE Transactions on Automatic Control, to appear, 2009.

6. Mason O., Shorten, R. Quadratic and Copositive Lyapunov Functions and the Stability of Positive Switched Linear Systems. In proceedings of American Control Conference, New York, 2007.

7. Mason O., Shorten R. On the Simultaneous Diagonal Stability of a Pair of Positive Linear Systems. Linear Algebra and its Applications, 413:13-23, 2006.

8. Berman A., Plemmons R. J. Non-negative Matrices in the Mathematical Sciences. SIAM Classics in Applied Mathematics, 1994.

9. Camlibel M. K., Schumacher J. M. Unsolved Problems in Mathematical Systems and Control Theory, chapter "Copositive Lyapunov Functions". Princeton University Press, http://press.princeton.edu/math/blondel, 2004.

10. Song, Y., Seetharama-Gowda, M., Ravindran, G. On Some Properties of P-matrix Sets. Linear Algebra and its Applications, 290:237-246, 1999.

11. Mason O., Shorten R. On Linear Copositive Lyapunov Functions and the Stability of Switched Positive Linear Systems. IEEE Transactions on Automatic Control, 52(7):13461349, 2007. 